\title{
Comparison of nasopharyngeal and guttural pouch specimens to determine the optimal sampling site to detect Streptococcus equi subsp equi carriers by DNA amplification
}

\author{
Ashley G. Boyle ${ }^{1 *}$, Darko Stefanovski ${ }^{1}$ and Shelley C. Rankin ${ }^{2}$
}

\begin{abstract}
Background: Streptococcus equi subsp equi (S. equi) is the cause of "equine strangles" which is a highly infectious upper respiratory disease. Detection of $S$. equi is influenced by site of specimen collection, method of sampling, and type of diagnostic test that is performed. We hypothesized i) that a loop-mediated isothermal amplification (LAMP) assay that targets the $S$. equi-specific eqbE gene would be more sensitive than a realtime PCR assay that targets the S. equi-specific seel gene and ii) that LAMP of specimens obtained by guttural pouch lavage (GPL) would be more sensitive than LAMP of nasopharyngeal specimens to identify S. equi carriers.
\end{abstract}

Methods: A nasopharyngeal flocked swab, nasopharyngeal wash, and GPL specimen was collected from 44 convalescent horses and the eqbE LAMP assay was performed. The seel realtime PCR assay and aerobic culture were also performed on the GPL specimen. Logistic regression was performed to compare sampling sites and test methods ( $P$-values $\leq 0.05$ were considered significant).

Results: One of 41 nasopharyngeal flocked swabs, 6/38 nasopharyngeal wash and 24/44 GPL specimens were positive by eqbE LAMP. 18/44 GPL specimens were positive by seel PCR and S. equi was isolated from 4/44 of these specimens. Detection of S. equi DNA was 51 times more likely from the GPL samples than nasopharyngeal samples (OR 51.0, $P<0$. 0001). When eqbE LAMP GPL samples were positive, it was eight times more likely that the guttural pouch had any abnormality on endoscopy (OR 8.2, P $\leq 0.005$ ), almost 20 times more likely that mild empyema was found (OR 19.7, $P \leq 0.002$ ), and eight times more likely that the Seel PCR was positive for $S$. equi DNA (OR 8.1, $P \leq 0.006$ ).

Conclusion: This study demonstrates that guttural pouch lavage specimens should be used to detect $S$. equi and that the eqbE LAMP assay was comparable to the seel PCR.

Keywords: Loop amplification, PCR, Flocked swab, Strangles, Streptococcus equi, Horse

\footnotetext{
* Correspondence: boylea@vet.upenn.edu

${ }^{1}$ Department of Clinical Studies New Bolton Center, School of Veterinary

Medicine, University of Pennsylvania, 382 West Street Rd, Kennett Square, PA

19348, USA

Full list of author information is available at the end of the article
} 


\section{Background}

Strangles, caused by Streptococcus equi subsp equi (S. equi), is a highly infectious upper respiratory disease that has a high morbidity rate and poses a high financial burden for the equine industry [1-3]. Up to $20 \%$ of recovered horses post-outbreak are persistent carriers of the organism in their guttural pouches [2, 4, 5]. Carrier animals serve as a reservoir for continued spread in the equine population. Bacterial culture and PCR of nasopharyngeal wash and guttural pouch lavage (GPL) specimens have been used to detect $S$. equi for diagnostic testing of clinical suspects and for the detection of carrier animals [2, 3, 5]. Bacterial culture has been documented to have low sensitivity when there are low numbers of $S$. equi [6]. PCR is highly sensitive and specific when the target DNA is present in the specimen. However, during the carrier state organisms may present at a very low number, may be shed intermittently or may be dead; yet their DNA will still be detectable by PCR. Practitioners must obtain multiple sequential samples from convalescing animals in order to ensure a $90 \%$ chance of true negatives before comingling with susceptible animals [5].

Detection of $S$. equi carriers is influenced by several factors that include: 1) site of specimen collection (rostral nasal passage, nasopharynx versus guttural pouch; 2) method of sampling (flocked swab, rayon swab, versus wash); 3) culture versus PCR, 4) target gene of the PCR; and 5) DNA amplification method [1, 6-8]. It is well established that $S$. equi is harbored in the guttural pouch and that there is intermittent shedding of organisms into the nasopharynx $[3,5]$. The use of flocked swabs during specimen collection has recently been implemented in human medicine because of greater recovery and elution of organisms for testing of both bacterial and viral diseases [9]. Sampling rostral nasal passages of horses with acute strangles infection with flocked nylon swabs did not statistically improve the detection of $S$. equi via PCR and bacterial culture [1]. Sampling the horse nasopharynx with flocked nylon swabs for the detection of S. equi has not been investigated.

The identification of a more specific gene target for $S$. equi detection by PCR is constantly under investigation due to the high level of genetic homology of $S$. equi with Streptococcus equi subsp. zooepidemicus and Streptococcus pyogenes $[8,10,11]$. In 2008 , the $e q b E$ gene was shown to be unique to $S$. equi and absent from $S$. zooepidemicus, thus providing a single specific gene target for real time PCR not previously available [11]. Loop-mediated isothermal amplification (LAMP) is a nucleic acid amplification method that is performed at a constant temperature and can detect target DNA in less than 30 min unlike traditional PCR methods that require thermal cycling [12]. LAMP has been used to detect $S$. equi in horses using both the $s e M$ gene [12] and the eqbE gene [13]; the latter assay was shown to be more specific [10].
The reality of strangles disease in the field is that horses are treated with antibiotics to treat the infection and prevent a carrier state as quickly as possible and allow the release of the animal from quarantine. If the eqbE LAMP assay is reliable for the detection of $S$. equi using "real world" samples from client-owned animals, then it may be possible to develop a point-of-care diagnostic assay in a hand-held device for stall-side diagnostic testing [14]. We hypothesized that sampling the guttural pouch would be more sensitive than sampling the nasopharynx to identify carriers of $S$. equi. We hypothesized that the eqbE LAMP assay would be more sensitive than the real time seeI PCR based on the higher specificity of the $e q b E$ gene [10] and higher efficiency of LAMP over realtime PCR [15]. The aim of this study was to compare nasopharyngeal and guttural pouch specimens to determine the optimal sampling site to detect Streptococcus equi subsp equi carriers. Two DNA amplification methods and two gene targets were compared.

\section{Methods \\ Horses}

This study was approved by the University of Pennsylvania's Institutional Animal Care and Use Committee Protocol \#805146. All owners had informed consent and agreed to the Widener-New Bolton Center (NBC) Privately - Owned Animal Protocol \#1311-1. This study was performed using naturally occurring and recovering cases of $S$. equi infection amongst New Bolton Center field service equine patients. Signalment was recorded. Horses were confirmed as previously infected with $S$. equi (nasopharyngeal wash or guttural pouch lavage $S$. equi positive on PCR or culture, or clinical signs consistent with $S$. equi infection in a confirmed S. equi outbreak), and recovered from clinically apparent disease for at least 2 to 3 weeks. Time from the start of clinical signs to sample collection was recorded. Antibiotic history was recorded. All horses were sedated with detomidine (Dormosedan, Zoetis, Florham Park, NJ) $0.015-0.025 \mathrm{mg} / \mathrm{kg}$ of body weight for the procedures and were administered one dose of flunixin meglimine (Banamine $^{\oplus}$, Merck Animal Health, Kenilworth, NJ) $1.1 \mathrm{mg} / \mathrm{kg}$ of body weight after the procedure as an analgesic and an anti-inflammatory agent. Samples were collected using 3 different sampling site strategies, and tested using 3 different $S$. equi identification methods.

\section{Samples}

Three samples were collected from each horse in the following order. A nasopharyngeal flocked nylon swab was performed using a $100 \mathrm{~mm}$ flocked swab (Floqswab ${ }^{\mathrm{Tm}}$, Copan Diagnostics, Murietta, CA) taped to a $63.5 \mathrm{~cm}$ uterine polyvinyl chloride pipette. The flocked swab was then removed from the pipette and placed in the tube and media of a culturette $\left(\mathrm{BBL}^{\mathrm{TM}}\right.$ CultureSwab ${ }^{\mathrm{TM}}$, Becton Dickinson, 
Franklin Lakes, NJ) for transport to the laboratory. Second, a nasopharyngeal wash was performed as previously described by the intranasal administration of $50 \mathrm{ml}$ of sterile saline [16], and third, an endoscopically-guided guttural pouch lavage was performed through a closed system of sterile polyethylene tubing passed through the instrument channel in the sedated, standing horse [17]. The endoscope was cleaned with chlorohexidine (Nolvasan Solution, Zoetis, Persippany, NJ), rinsed with sterile water, and disinfected for $10 \mathrm{~min}$ with ortho-phthalaldehyde solution (Cidex OPA, Advanced Sterilization Products, Johnson and Johnson, Switzerland) between each horse on the farm. The following tests were performed on the samples:

1) Nasopharyngeal flocked swab eqbE LAMP assay for S. equi

2) Nasopharyngeal wash eqbE LAMP assay for $S$. equi

3) Guttural pouch lavage (split into 3 aliquots)

a) S. equi culture

b) S. equi seeI PCR

c) S. equi eqbE LAMP assay.

Visual examination of the guttural pouch was performed at the time of sampling through the endoscope on an attached video screen. Briefly, the endoscope was passed via the ventral meatus of the nasal passage, into the nasopharynx, and through the guttural pouch openings sequentially [17].

\section{Diagnostic testing}

All diagnostic testing (S. equi culture and S. equi seeI PCR) was performed at the University of Pennsylvania NBC Clinical Microbiology Laboratory, which is an AAVLD accredited laboratory.

\section{seel PCR assay}

The seeI PCR assay has been previously validated [18]. The limit of detection [6] and its use in detection of $S$. equi in clinical nasopharyngeal wash samples and guttural pouch lavage samples from sick, convalescent, and asymptomatic horses [19] has been published by our laboratory. DNA was extracted from a $1 \mathrm{ml}$ aliquot of the guttural pouch lavage using PrepMan Ultra as described by the manufacturer (Applied Biosystems, Foster City, CA). The lavage fluid was centrifuged (Eppendorf Centrifuge Model 5417C, Germany) for $3 \mathrm{~min}$ at 20,000 $\times g$, and the pellet was resuspended in $100 \mu \mathrm{l}$ of PrepMan Ultra and boiled for $10 \mathrm{~min}$ at $100{ }^{\circ} \mathrm{C}$. The boiled extract was then diluted 1/100 in nuclease-free water prior to PCR (Fisher Scientific, Pittsburgh, PA) [6].

Realtime PCR was performed with the following primers and probe as previously described by our laboratory [18].
seeI-F 5'-CGGATACGGTGATGTTAAAGA -3'

seeI-R 5'-TTCCTTCCTCAAAGCCAGA-3'

seeI probe 5'-TTTGGCCGCTCCTCTAGATTTCAA-3'

The positive control was S. equi ATCC 33398 and positive and negative controls were run with each assay. Each PCR reaction consisted of $20 \mu \mathrm{l}$ of a commercial 1 mastermix (Quantifast Pathogen and Internal Control detection kit, Qiagen, Valencia, CA) plus $5 \mu \mathrm{l}$ of extracted DNA for a final concentration of $3 \mathrm{U}$ Taq, $200 \mu \mathrm{M}$ dNTPs, $4 \mathrm{mM} \mathrm{MgCl}_{2}$ and $25 \mathrm{mM}$ HEPES. Amplification began with $8 \mathrm{~min}$ at $95^{\circ}$ C, followed by 45 cycles of $20 \mathrm{~s}$ at $95^{\circ} \mathrm{C}$ and $60 \mathrm{~s}$ at $60^{\circ} \mathrm{C}$. No internal amplification control was included.

\section{Bacterial culture}

A culturette $\left(\mathrm{BBL}^{\mathrm{Tm}}\right.$ CultureSwab ${ }^{\mathrm{Tm}}$, Becton Dickinson, Franklin Lakes, NJ) was submerged in the vortexed fluid sample, plated on a blood agar plate (Columbia CNA (colistin, nalidixic acid), Becton Dickinson, Franklin Lakes, $\mathrm{NJ}$ ), aerobically incubated at $35{ }^{\circ} \mathrm{C}$ overnight, and read at 24 and $48 \mathrm{~h}$. Beta-hemolytic, organisms that were also catalase negative were subcultured to a blood agar plate and incubated at $35{ }^{\circ} \mathrm{C}$ overnight. Isolates were then identified biochemically using a commercial system. Sensititre panel (Trek Diagnostics). In addition to this, isolates were subcultured to Cystine Trypticase Agar (CTA) with lactose and CTA with sorbitol (Becton Dickenson, BBL ${ }^{\mathrm{m}}$ $\mathrm{CTA}^{\mathrm{Tw}}$ Medium) and incubated at $35^{\circ} \mathrm{C}$ overnight. Streptococcus equi subsp equi does not ferment sorbitol or lactose in contrast to Streptococcus equi subsp zooepidemicus. Presumptive Streptococcus equi subsp equi isolates were confirmed to be Lancefield group $\mathrm{C}$ using a commercial latex agglutination system. (Remel, Lenexa, KS)

\section{LAMP assay}

The S. equi eqbE LAMP assay was performed for research purposes at the Matthew J. Ryan Veterinary Hospital (Ryan VHUP) of the University of Pennsylvania Research Microbiology Laboratory [10]. Research personnel at Ryan VHUP were blinded to seeI PCR results obtained at NBC and had no information on the clinical status of the animal from which samples were obtained. The eqbE LAMP assay was performed using primers that were designed with the PrimerExplorer V4 Software (http://primerexplorer.jp/elamp4.0.0/index.html). The gene sequence used was based on Genbank Accession (http://www.ncbi.nlm.nih.gov/): AM909652, Streptococcus equi subsp. equi integrative conjugative element ICESE2, strain 4047. The analytical sensitivity of eqbE LAMP was determined by using a 10-fold serial dilution (ranging from $5 \times 10^{2} \mathrm{ng}$ to $5 \times 10^{-7} \mathrm{ng}$ ) of ATCC $33398 \mathrm{~S}$. equi subsp. equi genomic DNA (data shown in Additional file 1: Table S1). The eqbE LAMP was carried out in a reaction mixture of $25 \mu \mathrm{l}$ containing $0.4 \mu \mathrm{M}$ of each outer primer (F3, B3), $1.6 \mu \mathrm{M}$ of each inner primer (FIP, BIP), $0.8 \mu \mathrm{M}$ of loop primer 
(LoopF, LoopR), 1X Isothermal Master Mix (Pro-Lab Diagnostics, Round Rock, TX), and approximately 20100 ng of genomic DNA. DNA was extracted from a $1 \mathrm{ml}$ aliquot of the guttural pouch lavage as previously described above (seeI PCR assay). Amplification began with 30 min at $65{ }^{\circ} \mathrm{C}$ followed by $2 \mathrm{~min}$ at $80{ }^{\circ} \mathrm{C}$ to terminate the reaction. Positive and negative controls were genomic DNA from ATCC 33398 Streptococcus equi subsp. equi and reaction mixture without DNA, respectively.

eqbE F3 5'- CACATAAAACTACAGTACAAGGT- 3'

$e q b E$ B3 5' - GCGAGTATGAGTAATGCCA- 3'

$e q b E$ FIP 5'- TAAAGCTTTTTCCCAAGAAGCTTCT

GCTGGTGGTCAATTCTCT- 3'

eqbE BIP 5'- ATAGGGCTTGGGCTGATGTTAATGC

TAAAATAACAACGTGGC- 3'

eqbE Loop F 5'- GCGCTTGTCCAACCCGAATA- 3'

$e q b E$ Loop B 5'- AAATAGTTGAACGAGTTTGAGC GGT- 3'

The eqbE LAMP assay was tested for inclusivity with a panel of 20 well-defined S. equi subsp. equi isolates and showed $100 \%$ sensitivity and specificity. The analytical specificity (exclusivity) was determined using a panel of 14 strains: S. equi subsp. zooepidemicus, S. pneumoniae, $S$. agalactiae (2), Staphylococcus pseudintermedius, Staphylococcus aureus, Enterococcus faecalis, Klebsiella pneumoniae, E. coli, Salmonella typhmimurium, Haemophilus influenzae, Corynebacterium ulcerans and Campylobacter fetus subsp. venerealis.

\section{Guttural pouch endoscopy}

All horses had guttural pouch endoscopy performed. The presence or absence of empyema, defined as any gross purulent material in guttural pouch or within the lavaged fluid, in either guttural pouch was recorded. An endoscopic evaluation of "normal" or "abnormal" was recorded for the guttural pouches of each horse. An abnormal guttural pouch was defined when one or more of the following characteristics were found in either the left or the right guttural pouch: empyema, abnormal appearance to the respiratory epithelial lining, and/or visually enlarged retropharyngeal lymph nodes on the floor of the guttural pouch.

\section{Statistical analysis}

All statistical analysis was performed using computerized software (STATA 13.1, College Station, TX). Logistic regression was used to determine the odds ratio (OR) of detection of $S$. equi based on the sampling methods (nasopharyngeal flocked swab, nasopharyngeal wash, and guttural pouch lavage). The status of infection ( 0 , Negative; 1 , Positive) was determined using eqbE LAMP PCR. A $P$-value of $\leq 0.05$ was used to determine the significance of each statistical test. We adjusted for any potential confounding of the use of any antibiotic treatment (systemic or locally in the guttural pouch), the time from the last antibiotic treatment, and the number of days prior to eqbE LAMP testing that the sample was taken by determining their statistical significance in the logistic regression model [20].

\section{Results}

One hundred and twenty-three samples were analyzed (Additional file 2: Table S2): 41 nasopharyngeal flocked swabs, 38 nasopharyngeal washes, and 44 guttural pouch lavages were obtained from 40 different horses on 44 separate occasions from all 6 different strangles outbreaks that occurred from November 2013 to November 2014 (Fig. 1). On 3 occasions, the attending clinician neglected to obtain the nasopharyngeal samples and an additional 3 nasopharyngeal wash samples were lost

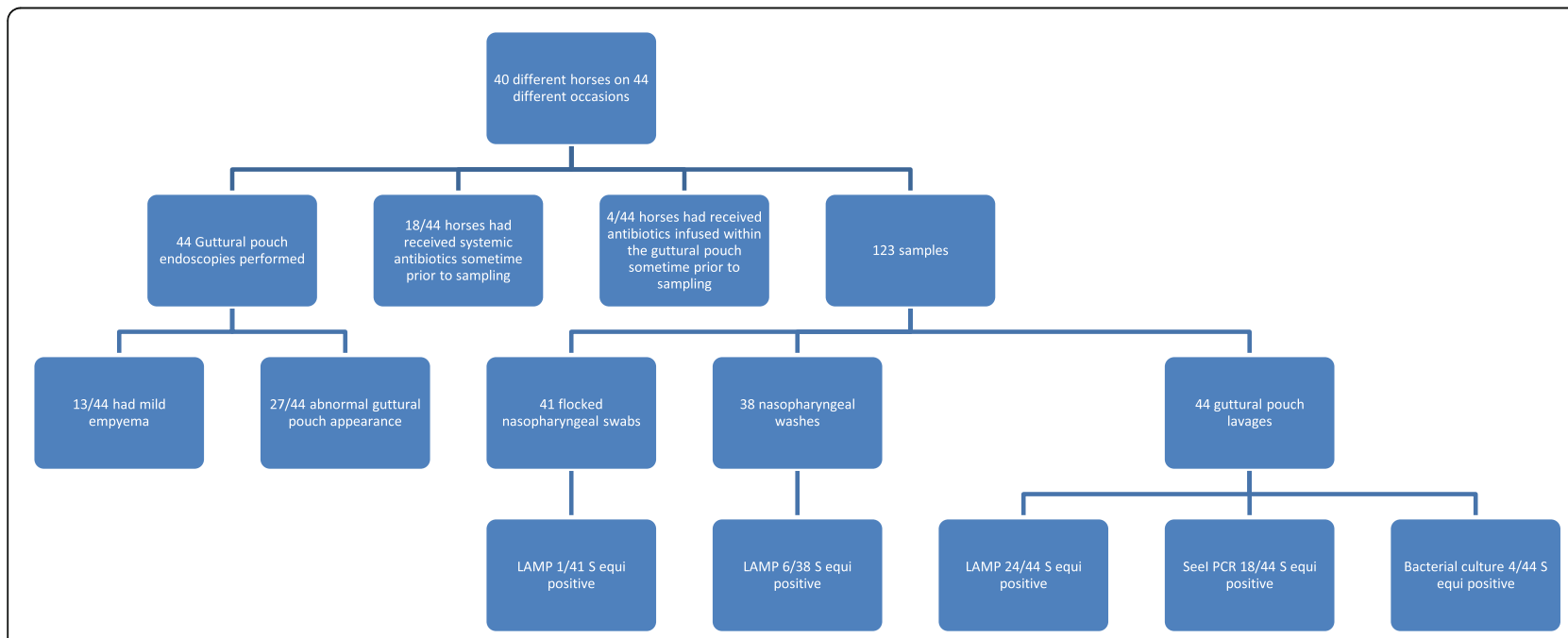

Fig. 1 Flow chart of antibiotic history, sample collection, diagnostics, results, and guttural pouchcharacteristics of 40 different S. equi convalescent horses obtained on 44 different occassions 
during transport to the research laboratory. Breeds consisted of 20 Standardbreds, 3 Quarter Horses, 9 Thoroughbreds, 2 Warmbloods, 5 draft horses, and one miniature horse. Seventeen horses were female, 16 were geldings, and 7 were male. The median age was 3 years (Interquartile Range [IQR] 1 to 3 years). The median time from start of clinical signs to sampling collection was 3 months (IQR 1 to 3 months).

A total of $31 / 123$ samples (41\%) were positive by eqbE LAMP: 1/41 nasopharyngeal flocked swab (2\%), 6/38 (16\%) nasopharyngeal wash and 24/44 (55\%) guttural pouch lavages. Eighteen of $44(41 \%)$ guttural pouch lavage samples were positive by seeI PCR. The median cycle threshold $\left(\mathrm{C}_{\mathrm{T}}\right)$ value was 34.32 (IQR 31.71-36.95). A seeI real-time PCR sample was considered negative if the $C_{T}$ value was $\geq 40$ cycles. S. equi was isolated via bacterial culture from 4/44 (9\%) guttural pouch lavage samples $[21,22]$, all of which were also positive by seeI PCR and $e q b \mathrm{E}$ LAMP. All horses that had a positive sample from the nasopharynx (either flocked swab or wash) were also positive in the guttural pouch. Seventeen (39\%) and 27 (61\%) of 44 horses had normal and abnormal endoscopic examinations of the guttural pouch, respectively. Thirtyone (70\%) of 44 horses had no empyema whereas 13 (30\%) of 44 horses had very mild empyema found on endoscopic examination of the guttural pouches. One horse required guttural pouch lavage to clear the particulate matter for clinical reasons. One of 44 horses was positive by seeI PCR and had a normal endoscopic examination with no empyema.

Eighteen of 44 horses $(41 \%)$ had a treatment history of systemic antibiotics: $12(67 \%)$ received procaine penicillin G (Agri-cillin, Zoetis, Florham Park, NJ), 4 (22\%) received ceftiofur (Excede, AgriLabs, St Joseph, MO), and 3 (17\%) received trimethoprim-sulfamethoxazole (Qualitest, Charlotte, NC). Four of the 44 horses (9\%) (4/18 [22\%] horses previously treated with systemic antibiotics) had a treatment history of antibiotic usage in the guttural pouch with penicillin-dihydrostreptomycin (Quartermaster, Pharmacia \& Upjohn Company, Kalamazoo, MI). In the 18 horses that were treated with antibiotics (systemic or locally within the guttural pouch), all antibiotic treatments finished a median of 3 weeks prior to sampling (IQR 1 week to 2.75 months). All samples were sent to Ryan VHUP and $e q b E$ LAMP testing was performed a median of 1 day after collection (IQR 1 day). The use of antibiotics and the number of days following their use that sample collection occurred could affect the positive or negative status of the sample. The number of days after sample collection the $e q b E$ LAMP testing was performed may affect the viability of the bacteria within the sample. All three independent variables were added to the 4 logistic models since they may confound the associations between eqbE LAMP and the outcomes.
The first logistic model estimated the association between eqbE LAMP and the location of sampling. When using the eqbE LAMP assay on 123 total samples, the guttural pouch lavage was 51 times more likely to be positive for $S$. equi DNA than the nasopharyngeal flocked swab and the nasopharyngeal wash samples (OR $51, \mathrm{P} \leq 0.0001$, 95\% CI 6.3-416.6). When the eqbE LAMP results of 44 guttural pouch lavage samples were positive, the guttural pouch was eight times more likely to have any abnormality on endoscopy (OR 8.2, P $\leq 0.005,95 \%$ CI 1.9-35.2), almost 20 times more likely to find mild empyema (OR 19.7, P $\leq 0.002$, 95\% CI 3.0-129.2), and the SeeI PCR was eight times more likely to be positive for $S$. equi DNA (OR 8.1, $\mathrm{P} \leq 0.006,95 \%$ CI 1.8-36.3). Table 1 shows the sensitivity, specificity, and receiver operator curves for all four logistic models. All models were normalized for the number of days before eqbE LAMP testing, antibiotic use, and time from last antibiotic treatment to sample collection.

\section{Discussion}

This study provides strong evidence that the eqbE LAMP assay performed with guttural pouch specimens is more sensitive for the detection of $S$. equi than nasopharyngeal flocked swab or a nasopharyngeal wash taken from the same horse at the same time. The 2005 American College of Veterinary Internal Medicine Consensus Statement recommendation for the detection of $S$. equi carriers is to obtain 3 negative nasopharyngeal washes or swab PCR samples over a 3 week period in order for an animal to be considered free of the organism. If the animal is found to be positive, endoscopic examination of the guttural pouch is strongly recommended [5]. The data presented here show that a single guttural pouch lavage nucleic acid amplification test in conjunction with visual examination of the guttural pouches in the convalescent period provides a cost and time efficiency to determine S. equi status of an animal

$e q b E$ LAMP was found to have acceptable discrimination when seeI PCR is considered as a true measure of outcome (ROC 0.78 ). The sensitivity and specificity of eqbE LAMP were lower when compared to the eqbE real time PCR results reported by North, $95 \%$ and $86 \%$, respectively [10]. This is also true when compared to the triplex quantitative PCR reported by Webb, et al. that reported a sensitivity of $94 \%$ and specificity of $97 \%$ [8]. These previous studies compared samples from horses at all stages of disease (acute, subacute, convalescent) and from all types of samples (abscess aspirates, rostral nasal swabs, nasopharyngeal swabs, nasopharyngeal washes and guttural pouch lavages) which inherently means that the bacterial counts (and therefore gene copies) varied from very high (samples with $10^{8}$ bacteria in the acute horses and abscess aspirates) to very low (potentially as low as 10 colony forming units in washes of convalescent horses) [6]. This study provides validation for the use of eqbE LAMP assay on the typical 
Table 1 Sensitivity and specificity comparing 1) 123 eqbE LAMP results to the sample type (guttural pouch lavage (GPL) versus not [nasopharyngeal wash, or nasopharyngeal flocked swab]); 2) eqbE LAMP results from 44 GPL samples to abnormal guttural pouch endoscopic findings; 3) eqbE LAMP from 44 GPL samples to empyema found on guttural pouch endoscopy; 4) eqbE LAMP to seel PCR from 44 GPL samples

\begin{tabular}{|c|c|c|c|c|c|c|c|c|c|c|}
\hline & & \multicolumn{2}{|c|}{ eqbE LAMP } & \multirow[t]{2}{*}{ Total } & \multirow[t]{2}{*}{ Sensitivity } & \multirow[t]{2}{*}{ Specificity } & \multirow[t]{2}{*}{$P P V^{b}$} & \multirow[t]{2}{*}{ NPV } & \multirow[t]{2}{*}{ Correctly Classified } & \multirow[t]{2}{*}{$\mathrm{ROC}^{\mathrm{d}}$} \\
\hline & & Positive & Negative & & & & & & & \\
\hline \multirow[t]{3}{*}{ GPL ${ }^{a}$ Sampling Method } & Yes & 24 & 7 & 31 & $77 \%$ & $78 \%$ & $55 \%$ & $91 \%$ & $78 \%$ & 0.83 \\
\hline & No & 20 & 72 & 92 & & & & & & \\
\hline & Total & 44 & 79 & 123 & & & & & & \\
\hline \multirow[t]{3}{*}{ Abnormal Guttural Pouch Endoscopy } & Yes & 20 & 7 & 27 & $74 \%$ & $70 \%$ & $80 \%$ & $63 \%$ & $72 \%$ & 0.80 \\
\hline & No & 5 & 12 & 17 & & & & & & \\
\hline & Total & 25 & 19 & 44 & & & & & & \\
\hline \multirow[t]{3}{*}{ Guttural Pouch Empyema } & Yes & 12 & 1 & 13 & $92 \%$ & $61 \%$ & $50 \%$ & $95 \%$ & $70 \%$ & 0.85 \\
\hline & No & 12 & 19 & 31 & & & & & & \\
\hline & Total & 24 & 20 & 44 & & & & & & \\
\hline \multirow[t]{3}{*}{ seel PCR } & Positive & 15 & 3 & 18 & $83 \%$ & $65 \%$ & $63 \%$ & $85 \%$ & $73 \%$ & 0.78 \\
\hline & Negative & 9 & 17 & 26 & & & & & & \\
\hline & Total & 24 & 20 & 44 & & & & & & \\
\hline
\end{tabular}

The cutoff probability for sample type is $\geq 0.5$

${ }^{a}$ Guttural Pouch Lavage

${ }^{\mathrm{b}}$ Positive predictive value (based on the detection of $S$. equi DNA and not on the presence of live organisms)

${ }^{\mathrm{c}}$ Negative predictive value

${ }^{\mathrm{d}}$ Receiver operator curve

All analyses are adjusted for antibiotics usage and the time from last antibiotic treatment by determining if they were significant confounders within the logistic regression via STATA

samples that are tested at the end of an outbreak to clear a patient/farm from quarantine, the guttural pouches of outwardly healthy convalescent horses that have very low bacterial counts but that could still be infective. The GPL culture that was performed adhered to the diagnostic lab protocol and a $10 \mu \mathrm{l}$ aliquot was plated to the CAN plate. This volume is considerably less than the $1 \mathrm{ml}$ that was centrifuged for DNA extraction and this could have affected the culture results for those samples (23) that were positive on DNA amplification and culture negative. An important incidental finding is that the culture data shows a massive die-off occurred in the guttural pouch in the interval between acute phase strangles and convalescence and is consistent with recent studies in the UK showing $S$. equi undergoes genetic decay in the guttural pouch including loss of genes necessary for virulence and infectivity [23, 24]. This study found flocked swab sampling of the nasopharynx to be a poor method for testing with the eqbE LAMP PCR to detect S. equi in outwardly healthy convalescent horses. This is in contrast to the improved bacterial recovery that has been found in human medicine sampling and laboratory processing when using flocked swabs [9]. Other veterinary researchers have found that the use of flocked nylon swabs did not improve the detection of $S$. equi by PCR and bacterial culture of the rostral nasal passages in acute strangles infection [1]. This is the first report showing the lack of performance of flocked swabs in the nasopharynx and the convalescent horse.
At the start of this study, the triplex PCR developed by Webb et al. [8] was published, but was not available for research use due to commercial patenting. seeI is a gene that encodes a superantigenic toxin [25] that is a virulence factor of S. equi [26]. Concerns with the seeI PCR include a potential lack of specificity between $S$. equi subsp equi and subsp zooepidemicus due to shared genes for superantigens that may lead to false positive results with some $S$. zooepidemicus strains [27]. In addition, S. equi isolated from some persistent carriers have been shown to have deletions in the variable region of the $s e M$ gene that leads to a false negative test result, although deletions of $e q b E$ have also been documented [23, 27]. We attempted to address these limitations by comparing the eqbE LAMP results to alternative phenotypic or clinical gold standards (empyema and other abnormalities found on guttural pouch endoscopy) that would make a clinician suspicious that a S. equi convalescent horse is still positive. Guttural pouch abnormalities including empyema were a good predictor of a positive eqbE LAMP assay result. Positive LAMP or PCR in normal guttural pouches may be the result of biofilm on the respiratory epithelium [28]

Ideally, performing seeI PCR on all 123 samples, including all the nasopharyngeal samples would have provided a larger number of samples in which to compare the tests, but was beyond the financial scope of this study. The study met its power calculation recommendations of 33 samples. We did not include the traditional swab as an additional 
sample collection method, but nasopharyngeal wash has already been shown to be more sensitive than the traditional nasopharyngeal swab [1]. It would have been ideal to compare 3 nasopharyngeal washes over 3 separate weeks in the same horse to one guttural pouch of that horse, but this would be difficult due to the prolonged sampling period and quarantine necessary for this infectious disease in client-owned animals. The current strangles consensus statement is currently under revision to reflect the research advances over the last 11 years. It is known that $S$. equi is intermittently shed from the guttural pouch into the nasopharynx [3]. Horses that have had 3 PCR negative consecutive nasopharyngeal samples have been shown to infect naïve herdmates [28]. Unfortunately, it is not yet possible to quantitatively predict the risk of extended survival of live $S$. equi in the abnormal empyemic guttural pouch. The purpose of this study was to examine the efficacy of eqbE LAMP in a "real world" situation during the aggressive sample collection time period (at the end of an outbreak on convalescent animals), which includes all the possible confounders associated with treating client-owned animals such as antibiotic treatments and time constraints on quarantines. The statistical analysis attempted to account for these confounders.

\section{Conclusions}

This study demonstrated the guttural pouch as the preferred anatomic site to sample to detect $S$. equi DNA in outwardly healthy convalescent horses using the eqbE LAMP assay. When used in conjunction with visual examination of the guttural pouch it provides evidence to eliminate the need for repeat testing with nasopharyngeal washes or swabs, thus saving time and money. Since the LAMP assay that targets the eqbE gene has acceptable agreement with the seeI PCR, further field verification of the eqbE LAMP in a pointof-care device [14] that will enable stall side testing performed by the field veterinarian is warranted.

\section{Additional files}

Additional file 1: Limit of detection for eqbE LAMP assay was determined via a "standard curve" as 0.005 ng of DNA using S. equi ATCC 33398. (DOCX $23 \mathrm{~kb}$ )

Additional file 2: Raw data of 123 samples (nasopharyngeal flocked swabs, nasopharyngeal washes, and gutturalpouch washes) from 40 different $S$. equi convalesecent horses tested for S. equi via Seel PCR and eqbE LAMP on 44 different dates. (XLSX 33 kb)

\section{Abbreviations}

$C_{T}$ : Cycle threshold; LAMP: Loop mediated isothermal amplification; NBC: New Bolton Center; Subsp: Subspecies

\section{Acknowledgements}

The authors would like to thank Kathleen Boyajian, She ri Young, and Lynn Stephens for their technical support.
Funding

Financial support for this study was graciously provided by the Boehringer Ingelheim 2013 Advancement in Equine Research Award and the Raymond Firestone Research Trust Grant.

Availability of data and materials

All data generated or analysed during the current study are included in this published article [and its supplementary information files].

\section{Authors' contributions}

$A B, S D$, and $S R$ designed the study. AB collected all the samples. SR performed laboratory procedures. AB collated all results. SD performed statistical analysis. AB, SD, and SR drafted the manuscript. All authors read and approved final manuscript.

\section{Competing interests}

The authors declare that they have no competing interests.

Consent for publication

Included in the consent to participate in the study.

\section{Ethics approval and consent to participate}

This study was approved by the University of Pennsylvania's Institutional Animal Care and Use Committee Protocol \#805146. All owners had informed consent and agreed to the Widener-New Bolton Center (NBC) Privately -

Owned Animal Protocol \#1311-1.

\section{Publisher's Note}

Springer Nature remains neutral with regard to jurisdictional claims in published maps and institutional affiliations.

\section{Author details}

'Department of Clinical Studies New Bolton Center, School of Veterinary Medicine, University of Pennsylvania, 382 West Street Rd, Kennett Square, PA 19348, USA. ${ }^{2}$ Department of Pathobiology, School of Veterinary Medicine, University of Pennsylvania, 3900 Spruce Street, Room 4036, Philadelphia, PA 19104, USA.

Received: 24 November 2016 Accepted: 4 March 2017

Published online: 23 March 2017

\section{References}

1. Lindahl S, Baverud V, Egenvall A, Aspan A, Pringle J. Comparison of sampling sites and laboratory diagnostic tests for S. Equi subsp. Equi in horses from confirmed strangles outbreaks. J Vet Intern Med. 2013;27:542-7.

2. Newton JR, Wood JL, Dunn KA, DeBrauwere MN, Chanter N. Naturally occurring persistent and asymptomatic infection of the guttural pouches of horses with Streptococcus equi. Vet Rec. 1997;140:84-90.

3. Newton JR, Verheyen K, Talbot NC, Timoney JF, Wood JL, Lakhani KH, et al. Control of strangles outbreaks by isolation of guttural pouch carriers identified using PCR and culture of Streptococcus equi. Equine Vet J. 2000;32: 515-26.

4. Sweeney CR, Benson CE, Whitlock RH, Meirs DA, Barningham SO, Whitehead SC, et al. Description of an epizootic and persistence of streptococcus equi infections in horses. J Am Vet Med Assoc. 1989;194:1281-6.

5. Sweeney C, Timoney JF, Newton JR, Hines MT. Review of streptococcus equi infections in horses: guidelines for treatment, control, and prevention of strangles. J Vet Int Med. 2005;19:123-34.

6. Boyle AG, Boston RC, O'Shea K, Young S, Rankin SC. Optimization of an in vitro assay to detect Streptococcus equi subsp. equi. Vet Microbiol. 2012;159: 406-10.

7. Gronbaek LM, Angen O, Vigre H, Olsen SN. Evaluation of a nested PCR test and bacterial culture of swabs from the nasal passages and from abscesses in relation to diagnosis of streptococcus equi infection (strangles). Equine Vet J. 2006;38:59-63.

8. Webb K, Barker C, Harrison T, Heather Z, Steward KF, Robinson C, et al. Detection of streptococcus equi subspecies equi using a triplex $\mathrm{PPCR}$ assay. Vet J. 2013;195:300-4.

9. Goldfarb DM, Slinger R, Tam RK, Barrowman N, Chan F. Assessment of flocked swabs for use in identification of streptococcal pharyngitis. J Clin Microbiol. 2009;47:3029-30. 
10. North SE, Wakeley PR, Mayo N, Mayers J, Sawyer J. Development of a real-time PCR to detect streptococcus equi subspecies equi. Equine Vet J. 2013;46:56-9.

11. Heather Z, Holden MT, Steward KF, Parkhill J, Song L, Challis GL, et al. A novel streptococcal integrative conjugative element involved in iron acquisition. Mol Microbiol. 2008;70:1274-92.

12. Hobo S, Niwa H, Oku K. Development and application of loop-mediated isothermal amplification methods targeting the seM gene for detection of Streptococcus equi subsp. equi. J Vet Med Sci. 2012;74:329-33.

13. North SE, Das P, Wakeley PR, Sawyer J. Development of a rapid isothermal assay to detect the causative agent of strangles. J Equine Vet Sci. 2012;32 Suppl 10:S54-5.

14. Liu C, Mauk MG, Hart R, Qiu X, Bau HH. A self-heating cartridge for molecular diagnostics. Lab Chip. 2011;11:2686-92.

15. Mori Y, Nagamine K, Tomita N, Notomi T. Detection of Loop mediated isothermal amplification reaction by turbidity derived from magnesium pyrophosphate formation. Biochem Biophy Res Commun. 2001;289:150-4.

16. Holland RE, Harris DG, Monge A. How to control strangles infections on the endemic farm. Proc 52nd Annu Meet AAEP. 2006;52:78-80.

17. Dallap Schaer B, Aldrich E, Orsini JA. Emergency diagnostic endoscopy. In: Orsini JA, Divers TJ, editors. Equine emergencies. 4th ed. St. Louis: Elsevier Saunders; 2014. p. 61-4.

18. Baverud V, Johansson SK, Aspan A. Real-time PCR for detection and differentiation of Streptococcus equi subsp. equi and Streptococcus equi subsp. zooepidemicus. Vet Microbiol. 2007;124:219-29.

19. Boyle AG, Rankin SC, Duffee L, Boston RC, Wheeler-Aceto H. Streptococcus equi detection polymerase chain reaction assay for equine nasopharyngeal and guttural pouch wash samples. J Vet Intern Med. 2016;30:276-81.

20. Hosmer DW, Lemeshow S. Assessing the fit of the model. In: Hosmer DW, Lemeshow S, editors. Applied logistic regression. 3rd ed. Hoboken: Wiley; 2013. p. 153-225.

21. Boyle AG, Stefanovski S, Rankin SC. ACVIM abstracts: determining optimal sampling site for streptococcus equi subsp equi carriers using a loopmediated isothermal PCR assay. J Vet Intern Med. 2015;29:1278,

22. Boyle AG, Stefanovski D, Rankin SC. 10th IEIDC abstracts: determining optimal sampling site for streptococcus equi subsp equi carriers using a loopmediated isothermal PCR assay. J Eq Vet Sci. 2016;39:S14.

23. Harris SR, Robinson C, Steward KF, Webb KS, Paillot R, Parkhill J, Holden MT, Waller AS. Genome specialization and decay of the strangles pathogen, Streptococcus equi, is driven by persistent infection. Genome Res. 2015;25:1360-71.

24. Holden MT, Heather Z, Paillot R, Steward KF, Webb K, Ainslie F, et al. Genomic evidence for the evolution of streptococcus equi: host restriction, increased virulence, and genetic exchange with human pathogens. PLoS Pathog. 2009;5:e1000346. doi:10.1371/journal.ppat.1000346.

25. Alber J, El-Sayed A, Lammler C, Hassan AA, Weiss R, Zscho M. Multiplex polymerase chain reaction for identification and differentiation of streptococcus equi subsp. Zooepidemicus and streptococcus equi subsp. Equi. Vet Med B Infect Dis Vet Public Health. 2004;51:455-8.

26. Waller AS. Strangles.taking steps towards eradication. Vet Micro. 2013;167:50-60.

27. Chanter N, Talbot NC, Newton JR, Hewson D, Verheyen K. Streptococcus equi with truncated M-proteins isolated from outwardly healthy horses. Microbiology. 2000;146:1361-9.

28. Waller AS. New perspectives for the diagnosis, control, treatment, and prevention of strangles in horses. Vet Clin North Am Equine. 2014;30:591-607.

\section{Submit your next manuscript to BioMed Central and we will help you at every step:}

- We accept pre-submission inquiries

- Our selector tool helps you to find the most relevant journal

- We provide round the clock customer support

- Convenient online submission

- Thorough peer review

- Inclusion in PubMed and all major indexing services

- Maximum visibility for your research

Submit your manuscript at www.biomedcentral.com/submit

C Biomed Central 